Bull. Austral. Math. Soc.

$13 \mathrm{G} 05,13 \mathrm{c} 05$

VOL. 60 (1999) [129-135]

\title{
QUASI-PROJECTIVITY OVER DOMAINS
}

\author{
DMitri Alexeev
}

\begin{abstract}
Let $R$ be an integral domain with quotient field $Q$. We investigate quasi- and $Q$ projective ideals, and properties of domains all ideals of which are quasi-projective. It is shown that the so-called $1 \frac{1}{2}$-generated ideals are quasi-projective, moreover, projective. A module $M$ is quasi-projective if and only if, for a projective ideal $P$ of $R$, the tensor product $M \otimes_{R} P$ is quasi-projective. Domains whose all ideals are quasiprojective are characterised as almost maximal Prüfer domains. $Q$ is quasi-projective if and only if every proper submodule of $Q$ is complete in its $R$-topology.
\end{abstract}

\section{INTRODUCTION}

Quasi-projective (sometimes called "self-projective") modules were introduced by Miyashita [7] as a generalisation of projective modules. These modules have been studied by a number of authors over a variety of rings. In particular, Herrmann [3] investigated quasi-projective modules over valuation domains. This paper contains generalisations of some of his results to arbitrary integral domains.

Let $R$ be an integral domain with quotient field $Q$. In the case of valuation domains, quasi-projectivity of an ideal is equivalent to its $Q$-projectivity. This does not seem to be true over general domains, although it holds if the ideal is finitely generated. $Q$ projectivity of ideals always implies their quasi-projectivity. We show that a submodule $V$ of $Q$ is quasi-projective if and only if $\operatorname{Ext}_{R}^{1}(V, U)=0$ for each submodule $U$ of $Q$.

For an ideal $I$ of $R$, this characterisation of quasi-projectivity reduces to vanishing $\operatorname{Ext}_{R}^{1}(I, J)$ for each ideal $J$ of $R$. This is used to identify the domains all of whose ideals are quasi-projective: these are exactly the almost maximal Prüfer domains. If we only assume the finitely generated ideals of $R$ to be quasi-projective, then $R$ becomes a Prüfer domain (not necessarily almost maximal).

Finally, we study the quasi-projectivity of $Q . Q$ is quasi-projective if and only if every proper submodule of $Q$ is complete in its $R$-topology.

Received 14th January, 1999

This paper is a part of the author's Ph.D. thesis written under supervision of Professor Laszlo Fuchs at Tulane University.

Copyright Clearance Centre, Inc. Serial-fee code: 0004-9729/99 \$A2.00+0.00. 


\section{Preliminaries}

Let $R$ be an integral domain, that is, a commutative ring with no zero divisors. Unless otherwise noted, we assume all modules to be unital $R$-modules, all ideals to be ideals of $R$, and all maps to be $R$-homomorphisms.

Definition. Let $M$ and $N$ be $R$-modules. $M$ is called $N$-projective or projective relative to $N$ if it is projective relative to all exact sequences of the form $0 \rightarrow N^{\prime} \rightarrow N \stackrel{\pi}{\rightarrow}$ $N / N^{\prime} \rightarrow 0$, where $N^{\prime}$ is a submodule of $N$ and $\pi$ is the canonical projection. That is, for every homomorphism $f: M \rightarrow N / N^{\prime}$ there exists a map $\bar{f}: M \rightarrow N$ such that the following diagram commutes:

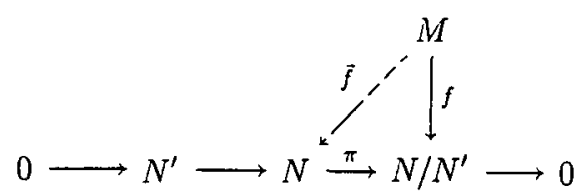

Thus, a module $M$ is projective if and only if it is $N$-projective for all $R$-modules $N$. The module $M$ is called self- or quasi-projective if it is $M$-projective.

We shall need the following lemma from Anderson and Fuller [1, p.188].

Lemma 1.1. Let $M$ be an $R$-module.

(a) If $0 \rightarrow A \rightarrow B \rightarrow C \rightarrow 0$ is an exact sequence of $R$-modules and $M$ is $B$-projective, then $M$ is projective relative to $A$ and $C$ as well.

(b) If $M$ is projective relative to $M_{i}$ for each $i$ in a finite index set $I$, then $M$ is $\bigoplus_{i \in I} M_{i}$-projective. Moreover, if $M$ is finitely generated, this holds for an arbitrary index set $I$.

The following corollary is a direct consequence of Lemma 1.1.

Corollary 1.2. Let $I$ be an ideal of domain $R$ and $V$ a submodule of $Q$.

(a) If $V$ is $Q$-projective, then $V$ is $U$-projective for every submodule $U$ of $Q$.

(b) If $I$ is quasi-projective, then $I$ is $J$-projective for every ideal $J$ of $R$.

Unlike projective ideals, quasi-projective ideals need not be finitely generated. In fact, Theorem 4.2 shows that if $R$ is an almost maximal Prüfer domain, then all ideals of $R$ are quasi-projective.

\section{Q-PROJECTIVITY}

We now turn our attention to $Q$-projectivity.

LEMma 2.1. Let $R$ be an integral domain. For a submodule $V$ of $Q$ the following statements are equivalent.

(a) $V$ is $Q$-projective. 
(b) $\operatorname{Ext}_{R}^{1}(V, U)=0$ for all submodules $U$ of $Q$.

(c) $V$ is $U$-projective for all submodules $U$ of $Q$.

Moreover, if $V=I$ is an ideal of $R$, then these conditions are equivalent to:

(d) For every submodule $U$ of $Q, Q / U$ has the injective property relative to the exact sequence $0 \rightarrow I \rightarrow R \rightarrow R / I \rightarrow 0$.

PROOF: $[(\mathrm{a}) \Leftrightarrow(\mathrm{b})]$ By applying functor $\operatorname{Hom}_{R}(V, *)$ to the exact sequence

$$
0 \rightarrow U \rightarrow Q \rightarrow Q / U \rightarrow 0
$$

we obtain the exact sequence

$$
0 \rightarrow \operatorname{Hom}_{R}(V, U) \rightarrow \operatorname{Hom}_{R}(V, Q) \stackrel{p}{\rightarrow} \operatorname{Hom}_{R}(V, Q / U) \rightarrow \operatorname{Ext}_{R}^{1}(V, U) \rightarrow \operatorname{Ext}_{R}^{1}(V, Q)=0
$$

Here $\operatorname{Ext}_{R}^{1}(V, U)=0$ if and only if $p$ is an epimorphism.

$[(\mathrm{a}) \Leftrightarrow(\mathrm{c})]$ This is Corollary $1.2(\mathrm{a})$.

$[(\mathrm{b}) \Leftrightarrow(\mathrm{d})]$ By applying the functors $\operatorname{Hom}_{R}(*, U)$ and $\operatorname{Hom}_{R}(R / I, *)$, respectively, to the exact sequences

$$
0 \rightarrow I \rightarrow R \rightarrow R / I \rightarrow 0 \text { and } 0 \rightarrow U \rightarrow Q \rightarrow Q / U \rightarrow 0
$$

we obtain the exact sequences

$$
\begin{gathered}
0=\operatorname{Ext}_{R}^{1}(R, U) \rightarrow \operatorname{Ext}_{R}^{1}(I, U) \rightarrow \operatorname{Ext}_{R}^{2}(R / I, U) \rightarrow \operatorname{Ext}_{R}^{2}(R, U)=0, \\
0=\operatorname{Ext}_{R}^{1}(R / I, Q) \rightarrow \operatorname{Ext}_{R}^{1}(R / I, Q / U) \rightarrow \operatorname{Ext}_{R}^{2}(R / I, U) \rightarrow \operatorname{Ext}_{R}^{2}(R / I, Q)=0 .
\end{gathered}
$$

It follows that $\operatorname{Ext}_{R}^{1}(I, U) \cong \operatorname{Ext}_{R}^{1}(R / I, Q / U)$. From the exact sequence

$$
\begin{aligned}
0 \rightarrow \operatorname{Hom}_{R}(R / I, Q / U) \rightarrow \operatorname{Hom}_{R}(R, Q / U) & \stackrel{p}{\rightarrow} \operatorname{Hom}_{R}(I, Q / U) \\
& \rightarrow \operatorname{Ext}_{R}^{1}(R / I, Q / U) \rightarrow \operatorname{Ext}_{R}^{1}(R, Q / U)=0
\end{aligned}
$$

we conclude that $p$ is epic if and only if $\operatorname{Ext}_{R}^{1}(R / I, Q / U)=0$.

\section{QUASI-PROJECTIVITY}

We begin this section by introducing a class of quasi-projective ideals.

Definition. An ideal $I$ of $R$ is called $1 \frac{1}{2}$-generated if it is two-generated, but one of the generators can be chosen to be any non-zero element of $I$. In other words, every proper homomorphic image of $I$ is a cyclic module. This is equivalent to saying that for any non-zero ideal $J<I$ we can find an $a \in I$ such that $I=R a+J$.

LEMMA 3.1. 1 $\frac{1}{2}$-generated ideals of a domain $R$ are quasi-projective. 
Proof: Let $I$ be a $1 \frac{1}{2}$-generated ideal. Suppose we are given a subideal $0 \neq K<I$, the canonical projection $\pi: I \rightarrow I / K$, and a homomorphism $f: I \rightarrow I / K$. To prove quasi-projectivity of $I$ we find a map $\bar{f}: I \rightarrow I$ such that $\pi \bar{f}=f$. The map $f$ factors as the canonical projection $\pi^{\prime}: I \rightarrow I / J$, where $J=\operatorname{Ker} f$, followed by an embedding $\varphi: I / J \rightarrow I / K$. The cyclic module $I / J$ is isomorphic to a submodule of cyclic module $I / K$. Therefore, there exists an $r \in R$ such that $r(I / K)=f(I)=\varphi(I / J)$. We claim that $\bar{f}: I \rightarrow I$ defined by $\bar{f}(t)=r t(t \in I)$ is a lifting of $f$ making the diagram

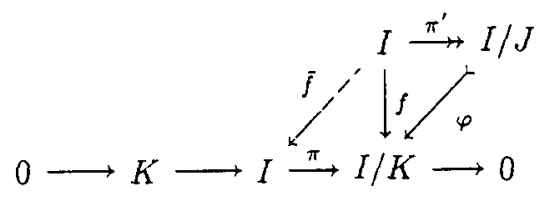

commutative.

To prove this claim, choose an $a \in R$ such that $I=R a+(K \cap J)$. Then $I=$ $R a+J=R a+K$. For the diagram to commute, it suffices to have $r J \subset K$. We have the following isomorphisms:

$$
\frac{I}{K}=\frac{R a+K}{K} \cong \frac{R a}{R a \cap K}, \quad f(I) \cong \frac{I}{J}=\frac{R a+J}{J} \cong \frac{R a}{R a \cap J}
$$

Now $r(I / K)=f(I)$ implies that

$$
\frac{r R a}{r R a \cap K} \cong \frac{R a}{R a \cap J} \cong \frac{r R a}{r R a \cap r J}
$$

We conclude that $r \operatorname{Ra} \cap K=r R a \cap r J$. It remains to note that

$$
J=(R a \cap J)+(J \cap K) \text { and } K=(R a \cap K)+(J \cap K)
$$

to conclude $r J=(r R a \cap r J)+r(J \cap K)=(r R a \cap K)+r(J \cap K) \subset K$. This completes the proof.

Rangaswamy and Vanaja [9] show that finitely generated torsion-free quasi-projective modules over a domain are projective. This immediately implies the following theorem.

THEOREM 3.2. $1 \frac{1}{2}$-generated ideals of any domain are projective.

The result also appears in [4] in a more general setting. Observe that over Prüfer domains of finite character, projective ideals are $1 \frac{1}{2}$-generated.

THEOREM 3.3. Let $P$ be a non-zero projective ideal of a domain $R$ and $M$ an $R$-module. $M$ is quasi-projective if and only if $P \otimes_{R} M$ is quasi-projective.

Proof: Suppose that $P \otimes_{R} M$ is quasi-projective. For a given map $f: M \rightarrow M / N$ $(N \subset M)$, we need to find a lifting $f^{\prime}: M \rightarrow M$. Tensoring with $P$ preserves exact sequences. The quasi-projectivity of $P \otimes_{R} M$ implies that there exists a map $\varphi: P \otimes_{R} M \rightarrow$ $P \otimes_{R} M$ such that the following diagram is commutative. 


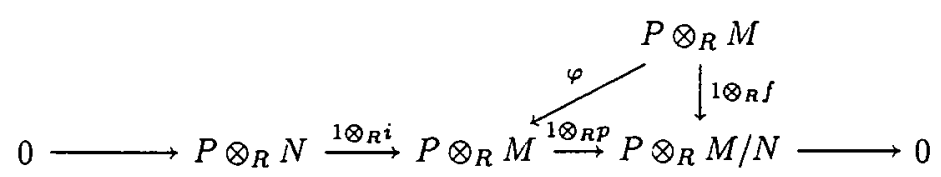

To show that $\varphi$ induces a map $f^{\prime}: M \rightarrow M$, we tensor the above diagram with the inverse $P^{-1}$ of $P$. Since $P^{-1} \otimes_{R} P \cong R$ and $R \otimes_{R} M \cong M$ for any $R$-module $M$ naturally, the map $\bar{f}=1_{P^{-1}} \otimes_{R} \varphi: M \rightarrow M$ is a desired lifting for $f$.

Conversely, suppose that $M$ is a quasi-projective module. We have

$$
P^{-1} \otimes_{R}\left(P \otimes_{R} M\right) \cong M,
$$

where $P^{-1}$ is projective. By the first part of the proof, this implies that $P \otimes_{R} M$ is quasi-projective.

It is worth while mentioning a consequence of Theorem 3.3 to the class semigroup of $R$. This is the semigroup of isomorphy classes of fractional ideals where identity is represented by $R$ and whose operation is ideal multiplication. Isomorphy classes of invertible ideals form a subgroup, the class group, of this semigroup. Theorem 3.3 asserts that quasi-projective ideals form complete orbits in the ideal semigroup of $R$ under the action of the class group.

\section{Prüfer DomaINS}

We formulate a close analogue of Lemma 2.1. The proof is similar to the original and is omitted.

LEMMA 4.1. The following are equivalent for an ideal $I$ of $R$.

(a) $I$ is quasi-projective.

(b) $\operatorname{Ext}_{R}^{1}(I, J)=0$ for every ideal $J$ of $R$.

(c) For every ideal $J$ of $R, Q / J$ has the injective property relative to the exact sequence $0 \rightarrow I \rightarrow R \rightarrow R / I \rightarrow 0$.

Lemmas 2.1 and 4.1, allow us to obtain a characterisation of domains all of whose ideals are quasi-projective; this characterisation is similar to that of valuation domains obtained by Herrmann in [3]. He has shown that a valuation domain each of whose ideals is quasi-projective is almost maximal. We precede the results with necessary definitions.

Definition. Let $M$ be a module over a commutative ring $R$. $M$ is said to be semi-compact if every finitely solvable set of congruences

$$
x \equiv x_{\alpha}\left(\bmod M_{\alpha}\right)
$$

where $x_{\alpha} \in M$ and $M_{\alpha}$ are submodules of $M$ which are annihilators of ideals of $R$, has a simultaneous solution in $M . M$ is called linearly compact if every family of cosets of submodules of $M$ that has the finite intersection property has a nonempty intersection. 
Following Brandal [2], we call a domain $R$ maximal if every homomorphic image of $R$ is linearly compact, and almost maximal if every proper homomorphic image of $R$ is linearly compact.

THEOREM 4.2. For an integral domain $R$, all of the following are equivalent.

(a) $\operatorname{Ext}_{R}^{1}(I, U)=0$ for every ideal $I$ of $R$ and submodule $U$ of $Q$.

(b) $\operatorname{Ext}_{R}^{1}(I, J)=0$ for every pair $I, J$ of ideals of $R$.

(c) Every ideal of $R$ is $Q$-projective.

(d) Every ideal of $R$ is quasi-projective.

(e) Every epimorphic image of $Q$ is injective.

(f) $R$ is a Prüfer domain and every homomorphic image of $Q$ is semi-compact.

(g) $R$ is an almost maximal Prüfer domain.

Proof: First, (a) implies (b) and (c) implies (d) trivially. (a) $\Leftrightarrow$ (c) is the statement of Lemma 2.1. (b) $\Leftrightarrow$ (d) is Lemma 4.1. Lemma 2.1 and Baer's criterion prove (c) $\Leftrightarrow$ (e). Matlis [5, Theorem 5] implies (e) $\Leftrightarrow(f)$. Finally, Olberding [8] shows the equivalence of (b), (e), and (g).

\section{Quotient Fields}

In this section we generalise a characterisation of valuation domains with quasiprojective quotient fields to the case of arbitrary integral domains. Herrmann [3] shows that a valuation domain $R$ is complete in its $R$-topology if and only if $Q$ is quasiprojective. We show that the same holds in general, almost unchanged.

We observe that $Q$ is projective relative to any ideal $I$ of $R$, or, more generally, to any proper submodule of itself. In fact, $Q$ is divisible and $I / J$ is bounded for ideals $J<I$. The only map $f: Q \rightarrow I / J$ is the trivial one, which can obviously be lifted. Of course, this property is far from being a sufficient condition for $Q$ to be quasi-projective. EXAmPLE. Let $R$ be the ring of integers $\mathbb{Z}$. Then $Q=\mathbb{Q}$, the field of rationals. For the localisation $\mathbb{Z}_{p}$ of $\mathbb{Z}$ at a prime $p \neq 0$, we have $\mathbb{Q} / \mathbb{Z}_{p} \cong \mathbb{Z}\left(p^{\infty}\right)$. By the above remark, $\mathbb{Q}$ is $I$-projective for each ideal $I$ of $\mathbb{Z}$. Since there are only countably many maps $\bar{f}: \mathbb{Q} \rightarrow \mathbb{Q}$, but uncountably many endomorphisms $\varphi: \mathbb{Z}\left(p^{\infty}\right) \rightarrow \mathbb{Z}\left(p^{\infty}\right)$, we conclude that it is impossible to lift every $f: \mathbb{Q} \rightarrow \mathbb{Q} / \mathbb{Z}_{p}$ to a map $\bar{f}: \mathbb{Q} \rightarrow \mathbb{Q}$ such that $\pi \bar{f}=f$. Hence, $\mathbb{Q}$ is not quasi-projective.

Definition. We may consider an $R$-module $M$ as a topological module equipped with the R-topology, in which a subbase for the open neighbourhoods of zero is formed by submodules $r M(0 \neq r \in R)$. The $R$-topology is a uniform topology. An $R$-module $M$ is said to be $R$-complete if it is Hausdorff and complete in its $R$-topology.

THEOREM 5.1. For any domain $R$, the following statements are equivalent:

(a) $Q$ is quasi-projective. 
(b) $\operatorname{Ext}_{R}^{1}(Q, U)=0$ for every submodule $U$ of $Q$.

(c) Every proper submodule of $Q$ is complete in its $R$-topology.

$[(\mathrm{a}) \Leftrightarrow(\mathrm{b})]$ We may assume $U \neq 0, Q$. By applying the functor $\operatorname{Hom}_{R}(Q, *)$ to the exact sequence $0 \rightarrow U \rightarrow Q \rightarrow Q / U \rightarrow 0$, we obtain the exact sequence

$$
\operatorname{Hom}_{R}(Q, Q) \stackrel{p}{\rightarrow} \operatorname{Hom}_{R}(Q, Q / U) \rightarrow \operatorname{Ext}_{R}^{1}(Q, U) \rightarrow \operatorname{Ext}_{R}^{1}(Q, Q)=0 .
$$

Thus we see that $p$ is epic if and only if $\operatorname{Ext}_{R}^{1}(Q, U)=0$.

[(b) $\Leftrightarrow(\mathrm{c})]$ Matlis [6] shows that a reduced torsion-free $R$-module $M$ is $R$-complete if and only if $\operatorname{Ext}_{R}^{1}(Q, M)=0$. Letting $M=U$ completes the proof.

We conclude this section with the following theorem.

THEOREM 5.2. Let $R$ be an integral domain. If $Q$ is quasi-projective, then $Q / R$ is indecomposable.

Proof: Let $Q$ be quasi-projective such that $Q / R$ is decomposable. Then, there are submodules $A$ and $B$ of $Q$ properly containing $R$ such that $Q / R=A / R \oplus B / R$. Consider the projection $f: Q \rightarrow A / R \oplus B / R$ on the first summand. By assumption, there exists a lifting $f^{\prime}: Q \rightarrow Q$ of $f$; this must be a multiplication by some non-zero element $q \in Q$. If $\pi: Q \rightarrow A / R \oplus B / R$ denotes the canonical projection, then $f=\pi f^{\prime}$ means that $q B \subseteq R$. Thus, $B$ is a fractional ideal, and so is $A$. But then $Q=A+B$ is impossible, a contradiction.

\section{REFERENCES}

[1] F. Anderson and K. Fuller, Rings and categories of modules, (second edition) (Springer-Verlag, Berlin, Heidelberg, New York, 1992).

[2] W. Brandal, 'Almost maximal integral domains and finitely generated modules', Trans. Amer. Math. Soc. 183 (1973), 203-222.

[3] P. Herrmann, 'Self-projective modules over valuation rings', Arch. Math. 43 (1984), $332-339$.

[4] D.C. Lantz and M.B. Martin, 'Strongly two-generated ideals', Comm. Alg. 16 (1988), 1759-1777.

[5] E. Matlis, 'Injective modules over Prüfer rings', Nagoya Math. 15 (1959), 57-69.

[6] E. Matlis, 'Cotorsion modules', Mem. Amer. Math. Soc. 49 (1964).

[7] Y. Miyashita, 'Quasi-projective modules, perfect modules and a theorem for modular lattices', J. Fac. Sci. Hokkaido Univ. Ser. I 19 (1966), 86-110.

[8] B. Olberding, 'Almost maximal Prüfer domains' (to appear).

[9] K. M. Rangaswamy and N. Vanaja, 'Quasi projectives in Abelian and module categories', Pacific J. Math. 43 (1972), 221-238.

Department of Mathematics

Tulane University

New Orleans LA 70118

United States of America

e-mail: alexeev@math.tulane.edu 